\section{Imminent rheumatoid arthritis can be identified in primary care}

We thank Vega-Morales $e t a l^{1}$ for their interest in our proposed approach for identifying individuals at risk of rheumatoid arthritis (RA) in a primary care setting. ${ }^{2} 3$ The authors agree that primary care is usually the first point of contact for patients with RA when they initially develop symptoms. For this reason, they agree that general practitioners (GPs) are well placed to be involved in screening strategies to identify individuals at risk of progressing to RA. $^{1}$

Vega-Morales et al advocate the squeeze test (Gaenslen's compression test) as a screening tool to aid GPs in identifying at-risk individuals for onward referral to a rheumatologist. The rationale for the squeeze test is that compression of the metacarpophalangeal (MCP) joints evokes pain in an individual with active synovitis. We agree that the squeeze test is useful for identifying early arthritis, ${ }^{4}$ but its sensitivity is limited and it may need to be combined with other screening tests in order to be sufficiently discriminatory. ${ }^{5}$ Nonetheless, it is cheap, quick and easy to perform and therefore an appropriate test for the GP who suspects a patient may have developed RA. Automation of the squeeze test, as proposed by Vega-Morales et al, ${ }^{1}$ may indeed have a role in this setting. Whether the squeeze test is sensitive enough to identify subclinical synovitis detected by ultrasound will be an important question to address.

Despite its use in early arthritis, we would argue that the squeeze test is inappropriate for identifying at-risk individuals, including those with imminent, but not yet established, synovitis. This important group, who have risk factors for RA (including systemic autoimmunity) but crucially do not have arthritis, must be identified before synovitis develops in order to benefit from potential preventative intervention. In line with this, the EULAR taskforce definition for arthralgia suspicious for progression to RA is aimed at identifying homogeneous patient groups that are at risk of developing arthritis before they progress to RA. ${ }^{6}$

Testing for anti-cyclic citrullinated peptide (CCP) antibodies in individuals who present to their GP with a new musculoskeletal complaint can effectively identify individuals at high risk of developing RA, before the onset of synovitis, and without the need for specialist assessment. ${ }^{3}$ In those that have a positive anti-CCP test, further risk assessment using clinical, serological and imaging tests can then quantify the risk of progression to arthritis more accurately. ${ }^{7}$ Thus, at-risk individuals may be followed in longitudinal studies and further stratified for risk appropriate intervention. The cost-effectiveness of this approach will need to be determined, but must be considered in the correct context; it may be a key advance towards preventing a disease that is associated with considerable morbidity, treatment and societal costs. ${ }^{8}$

Vega-Morales et al's response highlights the changing paradigm of early RA. The concept of early disease is changing from early arthritis to pre-arthritis while the target changes from early remission to prevention. As such, novel screening approaches are required. These must effectively identify at-risk individuals prior to the onset of synovitis for bone fide prevention to be feasible.

Kulveer Mankia, ${ }^{1,2}$ Paul Emery ${ }^{1,2}$

${ }^{1}$ Leeds Institute of Rheumatic and Musculoskeletal Medicine, University of Leeds, Leeds, UK

${ }^{2}$ NIHR Leeds Musculoskeletal Biomedical Research Unit, Leeds, UK

Correspondence to Dr Kulveer Mankia, Leeds Institute of Rheumatic and Musculoskeletal Medicine, Chapel Allerton Hospital, Chapeltown Road, Leeds LS7 4SA, UK; k.s.mankia@leeds.ac.uk

Competing interests None declared.

Provenance and peer review Commissioned; internally peer reviewed.

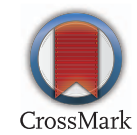

To cite Mankia K, Emery P. Ann Rheum Dis 2017;76:e42.

Accepted 9 February 2017

Published Online First 27 February 2017

\section{S Linked}

- http://dx.doi.org/10.1136/annrheumdis-2017-211205

Ann Rheum Dis 2017:76:e42. doi:10.1136/annrheumdis-2016-211231

\section{REFERENCES}

1 Vega-Morales D, Esquivel J, Arana-Guajardo A. Automated Squeeze test (Gaenslens maneuver) to identify suspicious arthralgia patients: Improving Time Delay to Rheumatology Consultation. Ann Rheum Dis 2017;76:e40.

2 Mankia K, Nam J, Emery P. Identifying arthralgia suspicious for progression to rheumatoid arthritis. Ann Rheum Dis 2017;76:e14.

3 Nam JL, Hunt L, Hensor EM, et al. Enriching case selection for imminent RA: the use of anti-CCP antibodies in individuals with new non-specific musculoskeletal symptoms-a cohort study. Ann Rheum Dis 2016;75:1452-6.

4 Emery P, Breedveld FC, Dougados M, et al. Early referral recommendation for newly diagnosed rheumatoid arthritis: evidence based development of a clinical guide. Ann Rheum Dis 2002:61:290-7.

5 van den Bosch WB, Mangnus L, Reijnierse M, et al. The diagnostic accuracy of the squeeze test to identify arthritis: a cross-sectional cohort study. Ann Rheum Dis 2015;74:1886-9.

6 van Steenbergen HW, Aletaha D, Beaart-van de Voorde LJ, et al. EULAR definition of arthralgia suspicious for progression to rheumatoid arthritis. Ann Rheum Dis 2017;76:491-6.

7 Rakieh C, Nam JL, Hunt L, et al. Predicting the development of clinical arthritis in anti-CCP positive individuals with non-specific musculoskeletal symptoms: a prospective observational cohort study. Ann Rheum Dis 2015;74:1659-66.

8 National Audit Office. Services for people with rheumatoid arthritis. 2009. http:/l www.nao.org.uk/report/services-for-people-with-rheumatoid-arthritis/ (HC 823 session 2008-2009) 